\section{MS18-04 Structures of meteoritic diamond nanocrystals}

Peter Nemeth ${ }^{1,2}$, Laurence A.J. Garvie ${ }^{3}$, Peter R. Buseck ${ }^{2,4}$

1. Institute of Materials and Environmental Chemistry, Research Center for Natural Sciences, Hungarian Academy of Sciences, H-1117 Budapest, Magyar Tudósok Körútja 2, Hungary.

2. School of Earth and Space Exploration, Arizona State University, Tempe, Arizona 85287-1404, USA

3. Center for Meteorite Studies, Arizona State University, Tempe, Arizona 85287-6004, USA

4. School of Molecular Sciences, Arizona State University, Tempe, AZ 85287-1604, USA

email: nemeth.peter@ttk.mta.hu

Meteoritic diamond nanocrystals can provide information on stellar nucleosynthesis ${ }^{1}$ as well as shock processes occurring during cosmic and terrestrial impacts $^{2}$. According to published data, these crystals are structurally inhomogeneous and consist of ordinary cubic (c-) diamond (space group: Fd-3m) plus a variety of $\mathrm{sp}^{3}$-bonded diamond polymorphs including $h$ (lonsdaleite), $i-, m$-, and $n$-diamond. These structures have received considerable attention because they are thought to indicate diagnostic formation conditions. In particular, the polymorphs have been widely used as indicators of asteroidal impacts and linked to mass extinctions such as the dinosaurs and mammoths. However, pure crystals, even tiny ones, of the polymorphs have never been reported. Furthermore, the diagnostic features of the polymorphs have been controversial, which posed serious problems with their identifications. In order to elucidate the structures of diamond nanocrystals, and thus to approach the issue of diamond polymorphs, we studied samples from the Canyon Diablo, Gujba, Murchison, and Orgueil meteorites as well as from the Popagai crater using an ultrahigh-resolution transmission electron microscope (uHRTEM). We found that diamond nanocrystals are intimately twinned and faulted. Combinations of $\{113\}$ and $\{111\}$ twins as well as $\{111\}$ stacking faults produce uHRTEM images and d-spacings that match those attributed to $h-, i-$, and $m$-diamond. The diagnostic features of $n$-diamond in TEM images can arise from crystal-thickness effects. Our data and interpretations strongly suggest that the reported diamond polymorphs are all actually $c$-diamond containing intimate twins and stacking faults ${ }^{3,4}$. This finding calls for reevaluation of implications regarding impact origins based on nanosized diamond polymorphs. The results also imply that defects are widespread in diamond nanocrystals, and these defects can give rise to a surprisingly diverse nanometer-scale structural complexity (Figure 1).

${ }^{1}$ Huss GR (2005) Meteoritic nanodiamonds: Messengers from the stars. Elements 1(2):97-100. ${ }^{2}$ Hough, RM, et al. (1997) Diamonds from the iridium-rich K-T boundary layer at Arroyo el Mimbral, Tamaulipas, Mexico. Geology 25: 1019-1022. ${ }^{3}$ Németh $\mathrm{P}$, et al. (2014) Lonsdaleite is faulted and twinned cubic diamond and does not exist as a discrete material. Nat. Commun. 5:5447. ${ }^{4}$ Németh P, Garvie LJA \& Buseck PR (2015) Twinning of cubic diamond explains reported nanodiamond polymoprhs. Sci. Rep. 5 (18381).

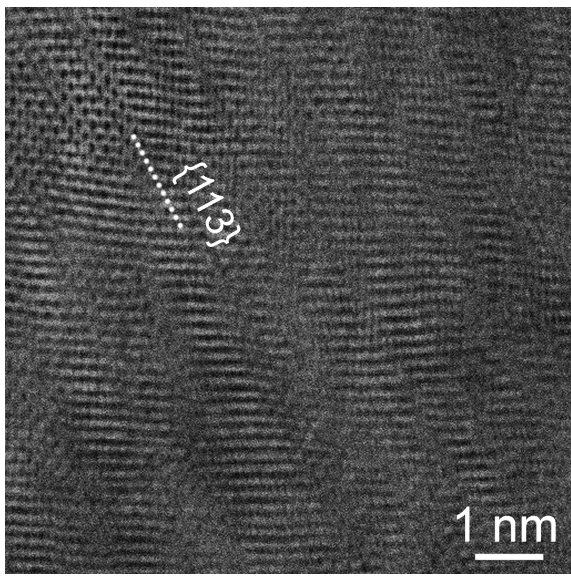

Figure 1. Structural complexity occurs in diamond as a result of $\{113\}$ twins. Sample: Canyon Diablo meteorite.

Keywords: diamond nanocrystals, diamond polymorphs, asteroidal impact, twins, stacking faults, structural complexity 